\title{
Knowledge, Attitude and Practices towards Hepatitis B Infection and Vaccination among Public Health Students in Ghana
}

\author{
Augustine Kumah ${ }^{*}{ }^{(0)}$, Emmanuel Tormeti², Gideon Dzando ${ }^{3}$, Hillary Selassi Nutakor ${ }^{4}$, \\ Wonder Andrea Yayra Amenuvor ${ }^{5}$, Charles Komla Anagblah6, Honore Mordenu', \\ Evans Awutey ${ }^{8}$, Hope Akpeke9, Prosper Kpobi10
}

\author{
${ }^{1}$ Department of Community Health, Ensign College of Public Health, Kpong, Ghana \\ ${ }^{2}$ Department of Nursing, Anfoega Catholic Hospital, Afoega, Ghana \\ ${ }^{3}$ Department of Nursing, Flinders University, Adelaide, Australia \\ ${ }^{4}$ Department of Mental Health, Accra Psychiatric Hospital, Accra, Ghana \\ ${ }^{5}$ Department of Oncology, Ghana Health Service, Ho, Ghana \\ ${ }^{6}$ Department of Nursing, Krachi West District Hospital, Krachi, Ghana \\ ${ }^{7}$ Department of Nursing, University of Cape Coast, Cape Coast, Ghana \\ ${ }^{8}$ Department of Pediatrics, Krachi West District Hospital, Krachi, Ghana \\ ${ }^{9}$ Department of Nursing, Jasikan District Hospital, Jasikan, Ghana \\ ${ }^{10}$ Department of Monitoring and Evaluation, Korle-Bu Teaching Hospital, Accra, Ghana \\ Email: *augustinekumah@gmail.com
}

How to cite this paper: Kumah, A., Tormeti, E., Dzando, G., Nutakor, H.S., Amenuvor, W.A.Y., Anagblah, C.K., Mordenu, H., Awutey, E., Akpeke, H. and Kpobi, P. (2021) Knowledge, Attitude and Practices towards Hepatitis B Infection and Vaccination among Public Health Students in Ghana. Open Journal of Preventive Medicine, 11, 43-53. https://doi.org/10.4236/ojpm.2021.111004

Received: December 18, 2020

Accepted: January 26, 2021

Published: January 29, 2021

Copyright $\odot 2021$ by author(s) and Scientific Research Publishing Inc. This work is licensed under the Creative Commons Attribution International License (CC BY 4.0).

http://creativecommons.org/licenses/by/4.0/

\begin{abstract}
Background: Hepatitis-B (Hep-B) infection is a global public health problem of great concern which affects more than $5 \%$ of the local population in Sub-Saharan Africa. This study assessed students' knowledge, attitude and practices towards Hepatitis B infection and vaccination at the University of Health and Allied Sciences, Ghana. Methods: A cross-sectional quantitative descriptive survey was conducted among 262 Public Health students of the University of Health and Allied Science of the Volta region of Ghana, using a multi-stage sampling technique in selecting participants. A structured questionnaire was used to collect data from the participants. Data was analyzed using SPSS Version 20.1. Results: Study respondents were mostly males, forming $69.8 \%$ as against $30.2 \%$ of their female counterparts. All (262) respondents had knowledge on Hep-B infection and the availability of a vaccine for preventing Hepatitis infection. However, despite their knowledge of existence, the majority (56.9\%) of the respondents felt they did not need to be protected from Hep-B infection. Majority (58.8\%) of the total respondents have been vaccinated against Hep-B infection. The study identified the cost of vaccines as a major setback to non-vaccination. However, out of the $41.2 \%$
\end{abstract}


respondents who were not vaccinated against Hep B infection, majority (50.9\%) of them were not willing to be vaccinated even if it was offered to them at no cost. Conclusion: There was a significantly high level of knowledge on Hep-B infection and the availability of vaccine for Hep-B infection among students. However, the majority of the respondents felt they did not need to be protected from Hep-B infection. More education is needed on the importance of vaccination as an effective measure for controlling Hepatitis infection.

\section{Keywords}

Knowledge, Practice, Attitude, Hepatitis B, Students, Ghana

\section{Introduction}

Hepatitis-B (Hep-B) infection is a global public health problem of great concern. The spectrum of the disease is highly variable ranging from mild disease to chronic liver diseases including hepatocellular carcinoma. There are approximately 350 million chronic Hepatitis-B surface antigen carriers in the world [1]. An estimated 257 million people are living with hepatitis B virus infection, in 2015 hepatitis B resulted in 887,000 deaths, mostly from complications including cirrhosis and hepatocellular carcinoma [2]. There is currently no permanent cure for Hepatitis Virus infection. Hence, prevention of the disease through vaccination is the only effective means to control the disease. Hepatis-B is caused by a DNA-virus, the Hepatitis-B Virus (HBV) [2]. The virus is highly contagious, 50 - 100 times more infectious than Human Immunodeficiency Virus (HIV), and is transmitted between people through blood, semen, vaginal fluids and mucous membranes, hence the most common ways of transmission are by unprotected sex, unsafe blood transfusions, unsafe use of needles, from mother to child at birth, close household contact and between children in early childhood [3].

Seven types of hepatitis virus have been identified; hepatitis A to $G$ and hepatitis $B$ remains the most serious type with high risk of death from liver cirrhosis and cancer. HBV is unique compared to other sexually transmitted diseases, because it can be prevented with vaccines [3]. Hepatitis B, C and D are transmitted by parenteral contact with infected body fluids including blood, semen, saliva, sweat and tears, invasive medical procedures using contaminated equipment, vertical transmission and from family member to child whereas hepatitis A, E, G are typically caused by ingestion of contaminated food or water, there are more than two billion people worldwide, having evidence of recent or past HBV infection and 350 million are chronic carriers [4]. According to the World Health Organization (WHO), HBV infection affects more than $5 \%$ of the local population in Sub-Saharan Africa and more than $8 \%$ in West Africa, reaching up to $15 \%$ in some areas [5]. The transmission of the virus occurs early in life and is associated with a low rate of spontaneous viral clearance and a high risk of 
chronic liver disease. It is estimated that $25 \%$ of young adults, infected during childhood, will die prematurely from HBV-related cirrhosis or Hepatocellular carcinoma (HCC) [6]. Immunization for HBV has been available since 1982 and has been shown to be highly effective in reducing the prevalence of hepatitis $B$ surface antigen (HBsAg) in children worldwide, moreover, national immunization programs have led to a substantial decrease in the burden of chronic liver disease and HCC in young adults born after 1984 [7].

HBV is an important occupational hazard for unvaccinated Healthcare workers (HCWs) including students, with an estimated 2 to 4 times higher incidence than the general population [8] [9]. Knowledge regarding the infection and its related safety precautions among HCWs is therefore essential to minimize the acquired infections in healthcare settings among this vulnerable population as they remain in direct contact with potentially infected persons, blood, and medical tools and instruments during the course of service delivery [10] [11]. Inadequate knowledge of HBV among HCWs however, may affect their behavioral pattern to vaccination and safety measures. In spite of the high burden of disease due to chronic Hepatitis B and the improvements and opportunities for care and treatment worldwide, the majority of people infected with HBV are unaware of their status and hence normally present with advanced stage of the disease [9]. In low-income countries, for instance, only about $5 \%$ of HBV infected persons know their infection status. This low uptake of HBV testing is a result of inadequate testing facilities or services, lack of effective policies and standards for testing, poor laboratory capacity and infrastructure, high cost and complex diagnostic assays and algorithms, and the use of substandard test kits and reagents [9].

The $\mathrm{HBV}$ vaccines (HBVc) have been in existence since 1992 and are available as monovalent formulations for birth doses or for vaccination of at-risk adult populations [12]. A standard three-dose vaccine regimen, with the second and third doses given 1 month and 6 months apart, respectively, from the initial dose, has been identified as very effective in conferring immunity against HBV [13]. Among HCWs and other healthy adults, HBV vaccination is known to be highly effective for prevention of the infection; hence HBV vaccination has been recommended by the WHO as a primary preventive strategy for the control of the infection among HCWs [14]. Many studies have estimated HBV knowledge and vaccination status among HCWs and medical students in many parts of the world [15]. Little evidence is however available in Ghana. This study therefore assessed students' knowledge, attitude and practices towards Hepatitis B infection and vaccination at the University of Health and Allied Sciences, Ghana.

\section{Methods}

A cross-sectional quantitative descriptive survey was conducted among $262 \mathrm{Pub}-$ lic Health Students of the University of Health and Allied Sciences (UHAS) in the Volta region of Ghana. The University is one of the public institutions in 
Ghana, established in 2012 to offer higher education to undergraduates and graduates in Health-related fields including medicine, Nursing, Midwifery, Pharmacy and other Allied Health Sciences. The University currently has six schools/colleges including the School of Public Health, where this study was conducted. The School of Public Health is located in Hohoe and currently trains undergraduates and graduates in the field of Public Health.

A multi-stage sampling method was used in this study. First, a stratified sampling technique was used to select the various public health programmes. A simple random sampling was then employed in selecting participants. A proportional allocation was made according to the population of students in each programme. The study was carried out using a structured pretested questionnaire. The questionnaire assessed the respondents' Socio-demographic Characteristics, Respondents' Knowledge on Hep-B Infection and Vaccination, Respondents' Attitude towards Hepatitis B Virus (HBV) infection and Vaccination and Respondents' Practice of Hep-B Vaccination. Students who were not present in school at the time of the survey were excluded from the study. Data was analyzed using SPSS Version 20.1.

\section{Results}

\subsection{Socio-Demographic Characteristics}

Males dominated the study (69.8\%) and majority (34.3\%) were between the age group of 20-25 years. Majority (88.2\%) were single, while (11.8\%) were married. Majority (75.6\%) of the respondents were Christians' religion and (24.4\%) belonged to the Islamic religion. A proportion (38.2\%) of respondents studied Public Health Disease Control, (34.4\%) Public Health Informatics and (27.4\%) Public Health Promotion (Table 1).

\subsection{Respondents' Knowledge on Hep-B Infection and Vaccination}

All (262) respondents had knowledge on Hep-B infection and the availability of vaccine for preventing Hep-B infection. Majority (92.7\%) of the respondents knew that Hep-B infection is caused by a virus while (7.3\%) of respondents said it is caused by Bacteria. While majority $(71.0 \%)$ of the respondents knew that Hep-B can be transmitted, a proportion (69.1\%) of the respondents indicated that Hep-B infection is transmitted through having unprotected sex, and (30.9\%) of the respondents indicated sharing needles as mode of transmission. Majority (62.2\%) of the respondents identified vaccination as a measure for preventing Hep-B infection compared to (22.5\%) avoiding multiple sexual partners and (15.3\%) proper disposal of sharps as ways of preventing Hep-B infection. More than half (52.7\%) of the respondents knew Hep-B infection is not curable (Table 2).

Findings from the study indicated that the majority (54.6\%) of the respondents understood how predisposed they were to Hepatitis B infection, whereas the rest $45.4 \%$ held a contrary view. Although, majority (56.9\%) of the respon- 
dents felt they do not need to be protected from Hepatitis-B infection, a marginal (3.4\%) of those respondents have been vaccinated. About the significance of vaccination, the majority (67.9\%) of the total respondents believed it was necessary to be vaccinated at some point in time against Hepatitis B infection. This however juxtaposes the majority (56.9\%) who felt there was no need to be protected from Hepatitis B infection. While (49.6\%) of the respondents knew their Hep-B status, about half (50.4\%) of the respondents did not know their Hep-B status. None of the respondents ever sustained a needle-stick injury (Table 3).

The study identified that, majority $(58.8 \%)$ of the respondents have been vaccinated against Hep-B infection, while (41.2\%) of them were not vaccinated. Of the $(58.8 \%)$ respondents who were vaccinated, all of them have completed their vaccination schedule. However, out of the respondents who were not vaccinated (50.9\%) of them did not believe in vaccination as an important preventive measure for Hep-B infection and will not succumb to being vaccinated even if vaccination was offered at no cost. A proportion (49.1\%) of the respondents who were not vaccinated cited the cost of vaccination as the reason for not being vaccinated and expressed willingness to be vaccinated if the cost of vaccination was entirely waived (Table 4).

Table 1. Socio-demographic characteristics of respondents $(n=262)$.

\begin{tabular}{|c|c|c|}
\hline \multicolumn{3}{|l|}{ Gender } \\
\hline - Male & 183 & 69.8 \\
\hline - Female & 79 & 30.2 \\
\hline \multicolumn{3}{|l|}{ Age (Years) } \\
\hline - $20-25$ & 90 & 34.3 \\
\hline - $26-30$ & 81 & 30.9 \\
\hline - $31-35$ & 57 & 21.8 \\
\hline - $36-40$ & 34 & 13.0 \\
\hline \multicolumn{3}{|l|}{ Religion } \\
\hline - Christianity & 198 & 75.6 \\
\hline - Islam & 64 & 24.4 \\
\hline - Traditional & 0 & 0 \\
\hline \multicolumn{3}{|l|}{ Marital status } \\
\hline - Single & 231 & 88.2 \\
\hline - Married & 31 & 11.8 \\
\hline \multicolumn{3}{|l|}{ Programme of Study } \\
\hline - BPH (Disease Control) & 100 & 38.2 \\
\hline - BPH (Informatics) & 90 & 34.4 \\
\hline - BPH (Health Promotion) & 72 & 27.4 \\
\hline
\end{tabular}


Table 2. Respondents' knowledge on Hep-B infection and vaccination.

\begin{tabular}{lcc}
\hline \multicolumn{1}{c}{ Variable } & Frequency $(\mathbf{n})$ & Percentage (\%) \\
\hline Have heard about HB infection $(\mathbf{n}=\mathbf{2 6 2})$ & 262 & 100 \\
- Yes & 0 & 0.0 \\
- No & 243 & 92.7 \\
\hline Organism that causes HB infection $(\mathbf{n}=262)$ & 19 & 7.3 \\
- Virus & 0 & 0.0 \\
- Bacteria & 0 & 0.0 \\
- Protozoa & & 71.0 \\
- Fungi & 186 & 29.0 \\
\hline Long-time infection of HB can be transmitted $(\mathbf{n}=262)$ & 76 & 0.0 \\
- Yes & 0 & \\
- No & & \\
\hline
\end{tabular}

Mode of transmitting HB infection $(n=262)$

- Having unprotected sex

181

- Sharing needles

- Contaminated food and water

- Respiratory droplets

- Contaminated blood

0

0.0

There is vaccination for HB infection $(n=262)$

- Yes

$262 \quad 100$

- No

- I don't know

0

0.0

Means of preventing HB infection (262)

- Avoiding drinking contaminated water 0.0

- Avoiding food not well cooked

- Vaccination

- Proper disposal of sharps

- Avoiding multiple sexual partners

22.5

HB infection is curable $(n=262)$

- Yes

- $\mathrm{No}$

- I don't know

0 
Table 3. Respondents' attitude towards HBV infection and vaccination.

\begin{tabular}{|c|c|c|}
\hline Attitude & Frequency (n) & Percentage (\%) \\
\hline \multicolumn{3}{|l|}{ You feel at risk by virtue of your work $(n=262)$} \\
\hline - Yes & 143 & 54.6 \\
\hline - No & 119 & 45.4 \\
\hline - I do not Know & 0 & 0.0 \\
\hline Need to be protected from HB infection $(n=262)$ & 113 & 43.1 \\
\hline - Yes & & \\
\hline - No & 149 & 56.9 \\
\hline - I do not know & 0 & 0.0 \\
\hline \multicolumn{3}{|l|}{ Know of your HB status $(n=262)$} \\
\hline - Yes & 130 & 49.6 \\
\hline - No & 132 & 50.4 \\
\hline \multicolumn{3}{|c|}{ Considered it necessary to receive HB vaccination $(n=262)$} \\
\hline - Yes & 178 & 67.9 \\
\hline - No & 84 & 32.1 \\
\hline \multicolumn{3}{|c|}{ Have sustained needle-stick injury in the ward $(n=262)$} \\
\hline - Yes & 0 & 0.0 \\
\hline - No & 262 & 100 \\
\hline - I don't remember & 0 & 0.0 \\
\hline
\end{tabular}

Table 4. Respondents' practice of $\mathrm{HB}$ vaccination.

\begin{tabular}{|c|c|c|}
\hline Practice & Frequency $(n)$ & Percentage $(\%)$ \\
\hline \multicolumn{3}{|l|}{ Have been vaccinated against HB infection $(n=262)$} \\
\hline - Yes & 154 & 58.8 \\
\hline - No & 108 & 41.2 \\
\hline - I do not remember & 0 & 0.0 \\
\hline \multicolumn{3}{|l|}{ Have completed HB vaccination schedule $(n=154)$} \\
\hline - Yes & 154 & 100 \\
\hline - No & 0 & 0.0 \\
\hline - I do not remember & 0 & 0.0 \\
\hline \multicolumn{3}{|c|}{ Reason for not vaccinating against HB infection $(n=108)$} \\
\hline - Non-accessibility of vaccine & 0 & 0.0 \\
\hline - Unawareness of vaccine's availability & 0 & 0.0 \\
\hline - Cost of vaccine & 53 & 49.1 \\
\hline - I do not believe in the importance of the vaccine & 55 & 50.9 \\
\hline - I doubt the efficacy of the vaccine & 0 & 0.0 \\
\hline \multicolumn{3}{|c|}{ Willingness to be vaccinated if it is offered for free $(n=108)$} \\
\hline - Yes & 53 & 49.1 \\
\hline - No & 55 & 50.9 \\
\hline
\end{tabular}




\section{Discussion}

In this study, we described the knowledge regarding Hepatitis B Virus (HBV) infection, its mode of transmission, and prevention among the Public Health undergraduate students. We also studied the students' HBV status and vaccination history of the students. The study found a significantly high level of Knowledge on Hep-B infection among the students as all 262 students had knowledge on Hep-B infection, its causes, mode of transmission and the availability of vaccine as an effective means of controlling Hep-B infection. The background of the respondents, being public health students explains this finding. Their knowledge on Hep-B infection and its cause might have emanated from lessons from their public health courses taught at school. This finding is similar to the findings of, [1] [16] [17] [18], which showed that $81 \%, 87 \%, 83.32 \%$ and $76.7 \%$ of the respondents respectively have had knowledge on Hep-B infection. It was not surprising to have found majority of the respondents knowing that Hep-B can be transmitted through unprotected sex, with a proportion of the respondent's haven indicated sharing needles as another mode of transmission. This finding is consistent with a study by [1], which asserted that more than half of the participants were aware that HBV can be transmitted via unprotected sex and contaminated blood.

Testing and vaccination against HBV infection is the gateway for access to both prevention and treatment services and is an essential component of an effective response to the hepatitis epidemic. Testing provides an opportunity to link people to interventions to reduce transmission, through counselling on risk behaviors and Vaccination. HBV vaccination has been recommended by the WHO as the primary prevention strategy for the infection among healthcare workers [14]. This study found that all respondents knew there is a vaccine for Hep-B infection and majority of them have identified vaccination as an effective measure for preventing Hep-B infection compared to a proportion of respondents who thought avoiding multiple sexual partners and proper disposal of sharps were the effective measure of preventing Hep-B infection. It was however surprising that, not all respondents have been vaccinated or knew of their Hep-B status even though the study found $100 \%$ knowledge of respondents on Hep-B Vaccine. About Half (50.4\%) of the respondents did not know their Hep-B status and only a little less than half (49.6\%) of the respondents knew their Hep B status. This finding seems to be in contradiction with the finding of [18], which indicated that $36 \%$ of the respondents did not know their HBV status.

Even though, majority of the respondents felt they were at risk of Hep-B infection by virtue of the nature of their work, a significant proportion (56.9\%) of the respondents felt they did not need to be protected against Hep-B infection. However, the majority $(58.8 \%)$ of the respondents were vaccinated against Hep-B infection, while (41.2\%) of them were not vaccinated. The study further found that, out of the total respondents who were not vaccinated, majority of them did not believe in vaccination as an important preventive measure for 
Hep-B infection. Public Health students work, as colleagues, with physicians and Nurses in caring for patients during their clinical attachments in the hospitals and are commonly exposed to sustained needle stick injuries during their clinical training, which may lead to serious or fatal infections with blood-borne pathogens such as HBV. This is why it is important to have public health students vaccinated against $\mathrm{HBV}$.

This study also identified the Cost of the Hep-B vaccine as a major reason for students not being vaccinated. Hepatitis B testing and vaccination in Ghana outside the Expanded Program on Immunization (EPI) are not covered under the National Health Insurance scheme and hence one has to pay more than a dollar before getting tested and has to pay more than $\$ 4.0$ to receive a single dose of Hep-B vaccine. Poor and incomplete Hep-B vaccine uptake among health workers and medical students have been reported by many studies [19] [20] [21], and the cost of vaccination were cited by most of these studies as the main reason for low uptake. Similarly, findings from [22] reported the cost of the vaccine, and the belief that vaccination is not an important preventive measure that to be taken as reasons for failure to be vaccinated. Surprisingly, the majority who were not vaccinated indicated that they will not be vaccinated even if it was offered for free. The fear of needle prick by most students might be the reason for the unwillingness to be vaccinated even if it was offered for free. This finding however contradicts the finding of [23] which affirmed that more than half of the unvaccinated nursing students were willing to be vaccinated if it was offered to them for free.

\subsection{Limitation}

The descriptive survey and the structured data collection instrument used in this study did not enable the participants to express themselves candidly, and thus limited the researchers' ability to probe. If qualitative aspects were included in this study, it could have been more efficient in exploring the respondents' view, deciphering other associated factors and to strengthen the findings of the quantitative study through in-depth interviews. Secondly, vaccination status was self-reported and not verified by vaccination records. So, recall bias could have led to over- or underestimation of coverage.

\subsection{Ethical Consideration}

Ethical approval for the study was obtained from the University of Health and Allied Science Ethical Review Board. However, local permission and approval for the study was required and obtained from the participants. Participation was voluntary and respondents could withdraw at any stage without any penalty.

\section{Conclusion}

There was a significantly high level of knowledge on Hep-B infection and the availability of vaccine for Hep-B infection. However, the majority of the respon- 
dents felt they did not need to be protected from Hep-B infection even though they believed they were at risk of contracting the infection. Surprisingly, most of the students who were not vaccinated against Hepatitis infection were not willing to be vaccinated even if it is offered for free. Cost of the vaccine was identified as a major reason for students not being vaccinated against Hep-B infection.

\section{Recommendation}

The Ministry of Health and the Health Training institutions must intensify education on the importance of vaccination as an effective measure for controlling Hepatitis infection. Secondly, the cost of Hepatitis B vaccination should be looked at and possibly made free for all health trainees to ensure a wider coverage of the vaccination amongst students. The researchers further recommend that vaccination against Hepatitis B should be made compulsory for all students in Health training institutions.

\section{Acknowledgements}

We would like to thank all the study participants and Lecturers of the University of Health and Allied Science (UHAS), who assisted with recruitment.

\section{Conflicts of Interest}

The authors declare no conflicts of interest regarding the publication of this paper.

\section{References}

[1] Johnson, D.W., et al. (2009) Frequencies of Hepatitis B and C Infections among Haemodialysis and Peritoneal Dialysis Patients in Asia-Pacific Countries: Analysis of Registry Data. Nephrology Dialysis Transplantation, 24, 1598-1603. https://doi.org/10.1093/ndt/gfn684

[2] WHO (2018) Hepatitis B Key Facts. World Health Organization, Geneva.

[3] Ul Haq, N., et al. (2013) A Cross-Sectional Assessment of Knowledge, Attitude and Practice among Hepatitis-B Patients in Quetta, Pakistan. BMC Public Health, 13, 448. https://doi.org/10.1186/1471-2458-13-448

[4] Malik, A.H. and Lee, W. (2000) Chronic Hepatitis B Virus Infection: Treatment Strategies for the Next Millennium. Annals of Internal Medicine, 132, 723-731. https://doi.org/10.7326/0003-4819-132-9-200005020-00007

[5] Ott, J.J., Stevens, G.A., Groeger, J. and Wiersma, S.T. (2012) Global Epidemiology of Hepatitis B Virus Infection: New Estimates of Age-Specific HBsAg Seroprevalence and Endemicity. Vaccine, 30, 2212-2219.

https://doi.org/10.1016/j.vaccine.2011.12.116

[6] WHO World Health Organization (2013) Hepatitis B, Fact Sheet No. 204.

[7] Chien, Y.C., Jan, C.F., Chiang, C.J., Kuo, H.S., You, S.L. and Chen, C.J. (2014) Incomplete Hepatitis B Immunization, Maternal Carrier Status, and Increased Risk of Liver Diseases: A 20-Year Cohort Study of 3.8 Million Vaccines. Hepatology, 60, 125-132. https://doi.org/10.1002/hep.27048

[8] Osei, E., Lokpo, S.Y. and Agboli, E. (2017) Sero-Prevalence of Hepatitis B Infection 
among Blood Donors in a Secondary Care Hospital, Ghana (2014): A Retrospective Analysis. BMC Research Notes, 10, Article No. 391.

https://doi.org/10.1186/s13104-017-2733-3

[9] WHO (2017) Guidelines on Hepatitis B and C Testing.

[10] West, D.J. (1984) The Risk of Hepatitis B Infection among Health Professionals in the United States: A Review. The American Journal of the Medical Sciences, 287, 26-33. https://doi.org/10.1097/00000441-198403000-00006

[11] Shiao, J., Guo, L. and McLaws, M.L. (2002) Estimation of the Risk of Bloodborne Pathogens to Health Care Workers after a Needlestick Injury in Taiwan. American Journal of Infection Control, 30, 15-20. https://doi.org/10.1067/mic.2002.119928

[12] Hou, J., Liu, Z. and Gu, F. (2005) Epidemiology and Prevention of Hepatitis B Virus Infection. International Journal of Medical Sciences, 2, 50-57. https://doi.org/10.7150/ijms.2.50

[13] Centers for Disease Control (2012) Updated CDC Recommendations for the Management of Hepatitis B Virus-Infected Health-Care Providers and Students. Morbidity and Mortality Weekly Report, 61, 1-12.

[14] World Health Organization (2016) Global Health Sector Strategy on Viral Hepatitis 2016-2021. The Global Hepatitis Programme Dep. HIV/AIDS.

[15] Darwish, M.A. and Al Khaldi, N.M. (2013) Knowledge about Hepatitis B Virus Infection among Medical Students in University of Dammam, Eastern Region of Saudi Arabia. Life Science Journal, 10, 860-867.

[16] Taylor, V.M., et al. (2006) Hepatitis B Knowledge and Practices among Chinese Immigrants to the United States. Asian Pacific Journal of Cancer Prevention, 7, 313-317.

[17] Maroof, K.A., Bansal, R., Parashar, P. and Sartaj, A. (2012) Do the Medical, Dental and Nursing Students of First Year Know about Hepatitis B? A Study from a University of North India. Journal of Pakistan Medical Association, 62, 25-27.

[18] Demetris, A.J., et al. (2016) Comprehensive Update of the Banff Working Group on Liver Allograft Pathology: Introduction of Antibody-Mediated Rejection. American Journal of Transplantation, 16, 2816-2835. https://doi.org/10.1111/ajt.13909

[19] Aroke, D., et al. (2018) Awareness and Vaccine Coverage of Hepatitis B among Cameroonian Medical Students. BioMed Research International, 2018, Article ID: 3673289. https://doi.org/10.1155/2018/3673289

[20] Ochu, C.L. and Beynon, C.M. (2017) Hepatitis B Vaccination Coverage, Knowledge and Sociodemographic Determinants of Uptake in High Risk Public Safety Workers in Kaduna State, Nigeria: A Cross Sectional Survey. BMJ Open, 7, e015845. https://doi.org/10.1136/bmjopen-2017-015845

[21] Biset Ayalew, M. and Adugna Horsa, B. (2017) Hepatitis B Vaccination Status among Health Care Workers in a Tertiary Hospital in Ethiopia. Hepatitis Research and Treatment, 2017, Article ID: 6470658. https://doi.org/10.1155/2017/6470658

[22] Bakry, S.H., Mustafa, A.F., Eldalo, A.S. and Yousif, M.A. (2012) Knowledge, Attitude and Practice of Health Care Workers toward Hepatitis B Virus Infection, Sudan. International Journal of Risk \& Safety in Medicine, 24, 95-102. https://doi.org/10.3233/JRS-2012-0558

[23] Mengal, H.R., Howteerakul, N., Suwannapong, N. and Rajatanun, T. (2008) Factors Relating to Acceptance of Hepatitis B Virus Vaccination by Nursing Students in a Tertiary Hospital, Pakistan. Journal of Health, Population and Nutrition, 26, 46-53. 